\title{
Diacronie
}

Studi di Storia Contemporanea

$N^{\circ} 23,3 \mid 2015$

Mediterraneo cosmopolita

\section{Nota introduttiva n. 23 - ottobre 2015}

\section{Luca Zuccolo}

\section{(2) OpenEdition}

\section{Journals}

\section{Edizione digitale}

URL: http://journals.openedition.org/diacronie/2354

DOI: 10.4000/diacronie.2354

ISSN: 2038-0925

\section{Editore}

Association culturelle Diacronie

\section{Notizia bibliografica digitale}

Luca Zuccolo, « Nota introduttiva n. 23 - ottobre 2015 », Diacronie [Online], № 23, 3 | 2015, Messo online il 29 octobre 2015, consultato il 24 septembre 2020. URL : http://journals.openedition.org/ diacronie/2354; DOI : https://doi.org/10.4000/diacronie.2354

Questo documento è stato generato automaticamente il 24 settembre 2020.

Creative Commons License 


\title{
Nota introduttiva n. 23 - ottobre 2015
}

\author{
Luca Zuccolo
}

Il Mediterraneo come spazio di comunicazione, come rete di relazioni ha svolto sempre un ruolo determinante nel confronto tra i diversi popoli cosmopoliti delle sue rive. Cultura, commerci, politica ed eserciti hanno svolto in questo panorama un ulteriore ruolo di collante e crescita sociale. Questo è il file rouge che lega i saggi di questo numero. Lo scambio culturale, infatti, ha molteplici forme che spaziano dalla diffusione di una lingua, trait d'union tra le diverse comunità mediterranee, sino al panorama dell'arte,

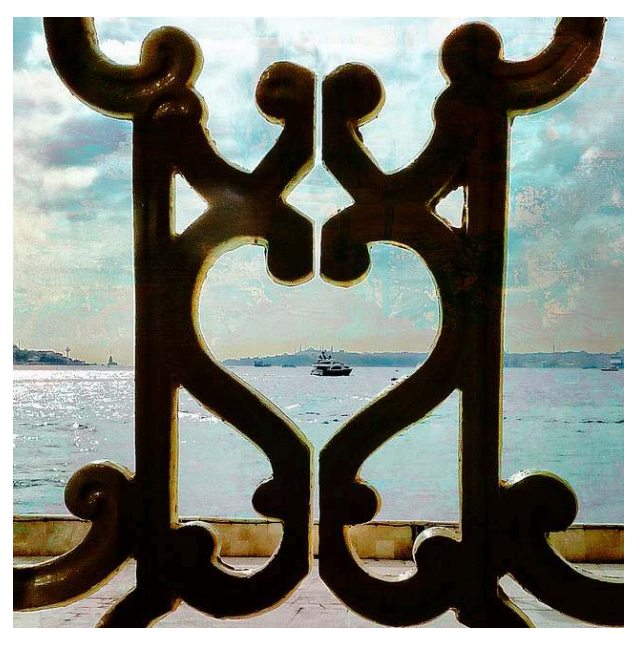
della letteratura e della satira. Ai due poli di questa comunicazione fittissima troviamo da un lato l'Impero ottomano, specchio sino al primo Novecento del cosmopolitismo mediterraneo, la Repubblica di Turchia suo epigono e suo malgrado protagonista di forzosi scambi culturali e, dall'altro lato, l'Europa nella sua plurima composizione - a sua volta cosmopolita - divenuta luogo di incontro, rifugio e scambio intellettuale.

2 Lo spazio mediterraneo, quindi, come spazio sociale e culturale in primis, ma anche terreno fertile per lo sviluppo di nuove forme di interrelazione. Un processo mai interrotto nonostante le guerre e la "costruzione" di confini, rigidi solo per chi li osserva dimenticando le proprie radici e le memorie condivise, ma pur sempre porosi alla condivisione di immagini e immaginari, di testi letterari e di opere artistiche.

Il Mediterraneo, quindi, è uno spazio della memoria stratificato sulle sue molteplici interrelazioni culturali che si intrecciano nel cosmopolitismo euro-ottomano prima, e poi nelle plurime relazioni sociali, politiche ed economiche del XX secolo. Un Secolo, 
quest'ultimo, che trova nel Mediterraneo - inteso come area geografica, come mare, ma anche come più ampia regione culturale e di memorie - il nuovo centro delle rotte migratorie, da secoli, frutto dei principali scambi socio-culturali che hanno fatto fiorire la regione ed oggi tragicamente note (sic!) per le troppo numerose vittime.

4 I saggi qui proposti forniscono uno spaccato di quello che si intende per interscambio e interrelazione nello spazio mediterraneo, uno spazio in cui la Turchia oggi e l'Impero ottomano prima hanno svolto un ruolo decisivo.

5 Una prima dimostrazione di questo è senza dubbio il saggio di Jonathan McCollum che descrive il processo di meticciato mediterraneo attraverso la visione nazionale e territoriale emersa sia in Italia che nell'Impero ottomano durante la guerra di Libia. La guerra, infatti, origina da entrambe le parti una nuova e dinamica definizione del proprio spazio territoriale e nazionale che vede le terre nord africane, poi divenute Libia, un importante tassello della propria identità e del loro spazio vitale.

6 Sulle memorie e sul loro legame con il territorio ritorna anche il saggio di Gökçe Bayındır Goularas. L'autrice propone tre temi cardine: il tema del confine, quello delle migrazioni e quello dell'identità. Temi che nel caso degli scambi forzosi di popolazione avvenuti tra Grecia e Turchia rappresentano il nodo centrale per una nuova categoria identitaria, quella di coloro che hanno dovuto attraversare un confine invisibile che si è concretizzato, soprattutto per loro, solo nel momento del passaggio e dello sradicamento. Nelle "storie" raccontate da Goularas, infatti, il confine diventa reale solo al momento del ricordo, della memoria degli affetti lasciati indietro, dall'altra parte. Il rapporto tra la propria identità interiore e la nuova identità è al centro del saggio qui presentato testimonianza di come i confini siano labili finché non si oltrepassano senza poter tornare indietro.

7 Infine, le miniere di Zonguldak e la peculiare struttura socio-culturale creatasi attorno a loro sono lo sfondo su cui Chantal Dennin-Lalart sviluppa la sua ricerca. Studiando le rappresentazioni culturali l'autrice descrive come una famiglia francese emigrata in Turchia per lavoro si sia adattata prima alla vita di miniera, poi alla vita turca e, infine, quali scompensi il rientro in patria abbia causato ai diversi membri del nucleo familiare.

8 Filo rosso il rapporto con l'altro e l'analisi del sé. Un sé che si trova, nel caso in oggetto, ad essere di volta in volta "altro" e "diverso" da chi lo circonda pur avendo esperito una crescita sociale e culturale. L'altro tema cardine del saggio, infatti, sono proprio i legami culturali, sociali ed economici da sempre presenti tra Turchi ed Europei che qui vengono riproposti dal case study in oggetto.

9 Il numero è completato da due recensioni tematiche che ben integrano i saggi presentati. La prima a cura di Matteo Anastasi presenta il volume di Bernardino Osio, diplomatico milanese, che racconta analizzando il carteggio del nonno materno Bernardino Nogara uno spaccato delle relazioni italo-ottomane all'inizio della Grande Guerra. La seconda, invece, curata da Luca Zuccolo, analizza il saggio di Erica Ianiro sui porti veneti nel mediterraneo orientale durante il XVIII secolo. Testimonianza, non solo delle strettissime relazioni tra la Porta Ottomana e la Serenissima, ma anche delle stratificazioni e delle interrelazioni mediterranee che da secoli influenzano e condizionano i diversi popoli che hanno abitato le rive del Mare Nostrum. 


\section{AUTORE}

\section{LUCA ZUCCOLO}

Dottore (PhD) in Storia Contemporanea del SUM di Napoli ha sviluppato una ricerca sulle dinamiche di formazione dell'identità ottomana e di un discorso di tipo patriottico durante il regno di Abdülhamid II (1880-1885) attraverso lo studio di fonti a stampa redatte in lingua francese. Già dottore magistrale in Storia d'Europa (Bologna 2008), si è occupato dello sviluppo della modernità durante l'ultimo secolo dell'Impero Ottomano, del confronto/scontro tra modernità e tradizione in un contesto cosmopolita e allo sviluppo dei movimenti sociali che hanno preparato l'avvento della società turca contemporanea. URL: <www.studistorici.com/ 2010/12/01/luca-zuccolo> 